\title{
O DOENTE MENTAL CRÔNICO INTERNADO: UMA REVISÃO DA LITERATURA
}

Zeyne Alves Pires Scherer ${ }^{1}$

Edson Arthur Scherer ${ }^{2}$

Scherer ZAP, Scherer EA. O doente mental crônico internado: uma revisão da literatura. Rev Latino-am Enfermagem 2001 julho; 9(4):56-61.

Abordagens alternativas no manejo da doença mental têm surgido no mundo inteiro com ênfase nos modelos extra-hospitalares, comunitários. Pouco se fala das pessoas cronicamente internadas. 0 objetivo da presente revisão da literatura foi levantar o que os estudiosos têm proposto na última década no que se refere ao trabalho com doentes mentais crônicos internados. Os artigos e textos encontrados foram divididos nos tópicos: características dos doentes crônicos internados, opinião destes sobre a internação e sugestões para melhorar a prática. Como conclusão, os autores propõem uma melhor avaliação com estudos sérios sobre esta clientela, a realidade que a cerca e as possibilidades de intervenção.

PALAVRAS CHAVE: pessoas mentalmente deficientes, tempo de internação, equipe de assistência ao paciente

\section{THE CHRONIC MENTALLY ILL PATIENT: A LITERATURE REVIEW}

Alternative approaches to manage mental illness have arisen all over the world with emphasis on outpatient community models. Little is said about chronically hospitalized patients. The purpose of this literature review was to access what researchers have proposed in the past decade concerning the work with chronic mentally ill inpatients. The articles found were divided in topics: characteristics of chronic mentally ill inpatients, their opinion about hospitalization and suggestions to improve the practice. As a conclusion, the authors propose a better assessment with serious studies concerning this clientele, their reality and the possibilities of intervention.

KEY WORDS: mentally ill persons, length of hospitalization, patient care team

\section{EL ENFERMO MENTAL CRÓNICO INTERNADO: UNA REVISIÓN DE LA LITERATURA}

Abordajes alternativos en el manejo de la enfermedad mental han surgido en el mundo entero con énfasis en los modelos extrahospitalarios, comunitarios. Poco se habla de las personas crónicamente internadas. El objetivo de la presente revisión de la literatura fue levantar lo que los estudiosos han propuesto en la última década acerca del trabajo con enfermos mentales crónicos internados. Los artículos y textos encontrados fueron divididos en los tópicos: características de los enfermos crónicos internados, opinión de los mismos sobre la internación y sugerencias para mejorar la práctica. Como conclusión, los autores proponen una mejor evaluación con estudios serios sobre esta clientela, la realidad que la circunda y las posibilidades de intervención.

PALABRAS CLAVES: personas con discapacidad mental, tiempo de internación, equipo de atención al paciente

\footnotetext{
${ }^{1}$ Enfermeira Psiquiátrica, Doutoranda da Área de Enfermagem Psiquiátrica e Especialista em Laboratório do Departamento de Enfermagem Psiquiátrica e Ciências Humanas da Escola de Enfermagem de Ribeirão Preto da Universidade de São Paulo, e-mai: scherer@eerp.usp.br; ${ }^{2}$ Professor da UNAERP, Mestre em Ciências Médicas da Faculdade de Medicina de Ribeirão Preto da Universidade de São Paulo, Médico Psiquiatra do Departamento de Neurologia, Psiquiatria e Psicologia Médica do HC-FMRP-USP
} 
INTRODUÇÃO

Mundialmente têm surgido abordagens alternativas para o manejo da doença mental. No entanto, os chamados casos crônicos de doença são colocados à margem, em relação aos agudos. Tem sido dada ênfase em tratamentos extra-hospitalares, comunitários e pouco se fala sobre os doentes ainda cronicamente internados. 0 fato da psiquiatria moderna, ter se tornado progressivamente "a histórica" ${ }^{\prime 1)}$, faz surgir a necessidade de reavaliar sua posição, começando por uma revisão da experiência histórica em psiquiatria.

As reformas psiquiátricas vêm sendo, inclusive, questionadas por serem vistas mais como movimentos políticos do que propriamente como sendo frutos da evolução e melhora da qualidade da assistência. Parece, pois, não ter havido com o advento dos serviços comunitários, uma redução significativa no uso dos leitos de internação psiquiátrica (sejam leitos em hospitais gerais como os de hospitais psiquiátricos públicos ou conveniados) e dos serviços de emergência, pelos doentes crônicos ${ }^{(2-5)}$. Os tratamentos destes se resumem, de forma geral, às abordagens psicofarmacológicas, aos manejos restritivos (contenção física, permanência em unidades fechadas), e à eletroconvulsoterapia (ECT), consideradas, muitas vezes, como abordagens iatrogênicas ${ }^{(2-3)}$.

Em uma pesquisa ${ }^{(6)}$ comparando as características de indivíduos internados em hospitais psiquiátricos tradicionais com os de instituições comunitárias (hospitais gerais, hospitais dia), foi concluído que não havia diferenças significativas entre estes grupos no que se refere a internações anteriores e comportamento dentro da instituição. No entanto, os admitidos nos hospitais tradicionais o eram, mais freqüentemente que o outro grupo, de forma involuntária; tinham indicações para serem internados que variavam de pressões familiares, de amigos, ou mesmo policial, por comportamento bizarro ou ameaçador; e viviam sob a dependência de cuidados básicos dos outros (família, sociedade). A rejeição( ${ }^{(7)}$, por sua vez, constitui-se em importante fator de desajuste social entre usuários de serviços de saúde mental e seus familiares, ou dos familiares entre si, o que contribui para uma difícil adaptação dos indivíduos à vida em comunidade.

Assim, através da presente revisão de literatura pretendemos destacar o que os autores têm proposto na última década no que se refere ao trabalho com doentes mentais crônicos internados. As fontes de referência foram: LILACS no período de 1983 até 1996; MEDLINE de 1986 até 1996; e PsycLIT de 1986 até 1996. Os resultados foram divididos nos seguintes tópicos: Características dos doentes crônicos internados; Opinião dos doentes sobre a internação; Sugestões para melhorar a prática.

\section{CARACTERÍSTICAS DOS DOENTES CRÔNICOS INTERNADOS}

É pouco provável que as pessoas cheguem ao hospital psiquiátrico por se sentirem infelizes ou por estarem sofrendo. Os usuários são levados, geralmente, quando sua conduta provoca inconvenientes, vergonha ou sofrimento aos outros, de forma que, ainda que o diagnóstico possa ser psiquiátrico, os sintomas estão relacionados à conduta social ${ }^{(7-9)}$.

As pessoas que permanecem por muito tempo internadas passam a apresentar mudanças em sua postura, com gestos típicos ("maneirismos") e acrescentam hábitos novos ao seu jeito de ser, como o de colecionismo e o de fumar. 0 colecionismo (ato de colecionar coisas, objetos) não se constitui necessariamente um sintoma patológico. Já o fumo é facilitado pela "atmosfera pró-fumo" à qual o indivíduo é exposto, visto que este é habitual nas instituições psiquiátricas hospitalares, tanto para usuários quanto para os técnicos que Ihes prestam assistência ${ }^{(10-11)}$.

Alguns doentes cronicamente hospitalizados são considerados como difíceis de tratar ${ }^{(12)}$. Estes são os que geralmente apresentam uma composição de dimensões diferentes de psicopatologia severa, ou seja, os que têm sintomas psicóticos persistentes, com alterações sérias de personalidade, com comportamento depressivo-suicida e agitações com violências.

Uma pesquisa sobre as características dos doentes internados, de 1970 a 1986, em hospitais psiquiátricos governamentais americanos, concluiu que houve mudanças no período estudado quanto às características dos distúrbios esquizofrênicos presentes ${ }^{(13)}$. Isto foi devido, provavelmente, as alterações feitas nas classificações dos distúrbios psiquiátricos, na prevalência da esquizofrenia e nos tipos de pessoas mais propensas a serem hospitalizadas. Quanto a este último aspecto, houve aumento no número de doentes considerados clinicamente e socialmente indigentes. Este achado, na opinião dos autores deste trabalho, aponta para a necessidade de melhor fundamentação e adequação dos hospitais estudados no sentido de oferecer assistência às necessidades desta nova clientela.

\section{OPINIÃO DOS DOENTES SOBRE A INTERNAÇÃO}

Os indivíduos internados em hospitais psiquiátricos são confrontados com complexos e, por vezes, conflituosos modelos e teorias sobre a causa e tratamento de suas doenças. Justificar-seia, portanto, a necessidade do usuário entender a proposta e o funcionamento da hospitalização, bem como é importante perceber as possiveis discrepâncias entre as perspectivas dos doentes e do hospital sobre os objetivos do tratamento ${ }^{(14)}$.

O sentimento de solidão entre doentes mentais hospitalizados foi tema de uma análise conduzida a partir da utilização de entrevistas semi-estruturadas gravadas em uma amostra de 12 pessoas internadas ${ }^{(15)}$. A categoria principal foi ilustrada pela metáfora 
"vivendo em uma ilha". Subcategorias incluíram tentativas de fuga e "sobrevivendo a ondas agitadas".

Em dois estudos conduzidos na Inglaterra, os autores ${ }^{(16-}$

17), utilizando método científico tentaram avaliar a satisfação dos doentes internados. Encontraram insatisfação por parte destes, expressas sob forma de queixas, como segue: "encontrando tantas pessoas mais doentes do que eu, me faz sentir pior"; pouca oportunidade de ver os médicos; estagiários de enfermagem não são maduros o suficiente para dar ajuda real; dormir e acordar muito cedo; poucas opções de espaço para receber as visitas; não oferecimento de cardápio variado às refeições; rádio e televisão constantemente ligados; decoração pobre; muitas ou poucas áreas para não fumantes; pouca informação sobre 0 tratamento e 0 funcionamento do serviço; medo em relação à segurança.

A reclusão utilizada em duas unidades de internação americanas, onde os doentes eram colocados em quartos fortes (sem mobília, com porta grossa de ferro com janela de vidro inquebrável, com paredes claras, com luminosidade e temperatura interna controlada por fora do quarto, com câmara de vidro no teto para facilitar o monitoramento pela enfermagem) foi pesquisada ${ }^{(18)}$ a partir da percepção das pessoas internadas. A cada 15 minutos eram feitas observações pelos técnicos, e a cada duas a quatro horas alguém entrava no quarto para prover assistência de higiene ao doente, medicamentos, sinais vitais, alimentos, ou para conversar. Os autores utilizaram um questionário previamente testado com enfermeiros e entrevistaram doentes selecionados pelas equipes como os que poderiam contribuir com o estudo. Como resultados, encontraram muitos sentimentos negativos sobre o processo de reclusão. A questão da condição humana, da dignidade, de entender e ser entendido, e de ser acolhido foram os temas prevalentes.

\section{SUGESTÕES PARA MELHORAR A PRÁTICA}

A eficácia dos neurolépticos na profilaxia de novos episódios agudos em doentes esquizofrênicos é irrefutável. Contudo, a medicação sozinha é incapaz de melhorar o grau de inserção dos usuários na comunidade. Tendo em vista este quadro, o tratamento deve ser planejado por longo prazo. Uma alternativa que tem sido muito valorizada é a dos programas de psicoeducação para os doentes (instruídos sobre sua doença e sobre a vida em comunidade pela equipe de assistência, principalmente psiquiatras, enfermeiros e terapeutas ocupacionais) e seus familiares (manejo da doença e dos familiares doentes, principalmente em trabalhos grupais com outras famílias coordenados por assistentes sociais) ${ }^{(19-22)}$.

Outra sugestão é a proposta de cada serviço avaliar a satisfação de seus doentes internados ${ }^{(16-17)}$. Nestes trabalhos, os autores concluem que seus achados não podem ser generalizados para outras instituições, mas que tais pesquisas são importantes fontes de dados para auxílio nas intervenções a serem planejadas em cada local específico, e que, portanto, estudos futuros na área são necessários.

Entre as sugestões para auxiliar os profissionais na tarefa de assistir aos internos crônicos de hospitais psiquiátricos quanto a suas necessidades sociais e clínicas, encontramos a proposta de dividir os doentes em dois grupos: um grupo dos que apresentam episódio agudo de doença no serviço, e o outro dos que têm enfermidade persistente de severidade contínua ou flutuante ${ }^{(23)}$. A finalidade de tal divisão seria a de proporcionar enfoque mais universal nas abordagens em cada grupo.

Dois pesquisadores propõem um modelo de psicoterapia breve orientado para soluções de problemas ${ }^{(24)}$, onde 0 usuário é levado a experienciar sua capacidade de lidar com dificuldades, de experimentar sua independência, e é estimulado a tomar suas próprias decisões sobre o que tem que enfrentar na vida.

Como as pessoas com doença mental são percebidas como portadoras de deficiências, o campo da reabilitação física pode ser usado como uma metáfora para a reabilitação psiquiátrica. A abordagem reabilitadora enfatiza 0 desenvolvimento de papéis $e$ capacidades de suporte necessárias para viver com sucesso, aprender e trabalhar na comunidade. Os componentes essenciais das técnicas de reabilitação psiquiátrica claramente são: a inserção de uma equipe de reabilitação dentro do contexto do hospital psiquiátrico; a definição dos papéis dos profissionais e o conhecimento fundamentado sobre como usar os papéis mais efetivamente; 0 acesso às capacidades funcionais e adaptativas dos doentes; tratamento psicofarmacológico; treinamento nos papéis sociais; reintegração ocupacional; e suporte familiar e da comunidade ${ }^{(25-27)}$.

Em uma análise sobre doentes mentais internados por longo prazo, foi observado que mesmo havendo redução em número de tais doentes através das intervenções na comunidade, estes eram substituídos por outros ${ }^{(28)}$. Sugerem os autores, que se há pretensão de reduzir no futuro o número de pessoas internadas a longo tempo, é necessário prosseguir no desenvolvimento e aprimoramento criativo de métodos de reabilitação e fazer estudos mais detalhados de seguimento de tal população que permanece internada apesar das tentativas de desinstitucionalizá-la, para que não seja considerada como grupo resistente a tal processo.

No que se refere especificamente ao trabalho de terapia ocupacional, sua importância no tratamento de doentes mentais crônicos, tem sido cada vez mais demonstrada, pois, com ela observase uma melhora na socialização, nos relacionamentos grupais, na aceitação da doença pelos usuários, que passam a buscar 0 tratamento, querem voltar ao trabalho, procuram por alguma atividade 
que os faça sentirem-se úteis, têm vontade de produzir e vencer a doença através da ocupação e mostram a necessidade de aprender alguma atividade artesanal ${ }^{(29-30)}$.

Outras modalidades de abordagem terapêutica têm sido testadas e sugeridas pelos autores. Entre elas aparece, por exemplo, a arte terapia ${ }^{(31)}$ que é feita de forma interdisciplinar, visando combater a dependência gerada pela institucionalização, explorando uma forma de terapia não verbal, mesmo que o trabalho se resuma somente em "fazer". Outra abordagem que precisa ser valorizada diz respeito ao lazer ou recreação, tida pelos doentes como importante por ajudálos a se distraírem, a relaxarem e a sentirem prazer ${ }^{(32)}$. Além destas, o uso terapêutico da música no ambiente tem sido explorado por ser uma área potencialmente rica, mas que necessita, ainda, de maiores investigações ${ }^{(33)}$.

Outra questão séria é o índice significativo de não adesão aos tratamentos por parte dos doentes mentais. Esta temática já foi bastante explorada na literatura especializada. Como alternativa, é sugerida a participação ativa do doente na administração da própria medicação durante a internação ${ }^{(34)}$. Segundo os autores, observandose o envolvimento no tratamento enquanto internado, é possível criar uma intervenção compreensiva para perceber o risco futuro de não adesão, e avaliar a efetividade da intervenção. A maioria dos internos que participaram do programa cometeu erros em poucos dias no controle da própria medicação, ou seja, em seguir a prescrição médica, demonstrando ser este um fator preditor de abandono de seguimento na alta, segundo os autores. Mesmo nestes casos, o programa ofereceu dados importantes que influenciaram decisões futuras sobre 0 tratamento.

Considerando que há indícios na literatura de que a psicose manifesta aumento em doentes internados que recebem café cafeinado após um período no qual receberam café descafeinado, foi conduzida uma pesquisa onde se removeu a venda de café e bebidas com cafeína em um hospital estatal de 1200 leitos $^{(35)}$. Observou-se a necessidade de reclusão e contenção dos doentes nos quatro meses seguintes, comparando os resultados com os 21 meses anteriores em que houve venda de cafeinados. As agressões contra outros doentes e membros da equipe reduziram em cerca de 25,0 \% e e incidência de destruição de objetos (patrimônio), reduziu em $51,0 \%$. Fica, assim, a proposta de reduzir a oferta de cafeinados aos usuários crônicos internados, como forma de diminuir a freqüência de episódios agressivos por parte destes.

Desde que o movimento de desinstitucionalização teve início, o modelo de serviço para os doentes crônicos tem sido caracterizado primeiro pelo mero número de locais para atendimento e, então, pela continuidade de cuidados conforme coordenado pelo sistema de manejo de caso ${ }^{(36)}$. Isto tem deixado escapar um elemento crucial para o sucesso do movimento, que é a manutenção da equipe de cuidados, ou seja, que uma mesma equipe básica de tratamento contínuo seja responsável pelos usuários, tanto no ambiente intrahospitalar quanto no extra-hospitalar.

As atitudes da equipe hospitalar para com os doentes mentais têm sido investigadas, descritas na literatura e discutidas em conferências. Tem sido sugerido que atitudes são precursoras ou determinantes de comportamentos. Enquanto normas e procedimentos hospitalares podem prescrever comportamentos da equipe, atitudes não podem ser ditadas. Frente a isto, pesquisadores sugeriram um programa através do qual é simulado um jogo entre os membros da equipe ${ }^{(37)}$. Tal atividade ficou conhecida como "um dia na vida de um doente internado", onde é proporcionado um experimento situacional para a equipe sobre a experiência da hospitalização vivenciada pelos doentes. O objetivo deste modelo é despertar a equipe quanto às suas atitudes frente aos usuários.

Estudando o hábito de fumar nas equipes de assistência e usuários de três hospitais psiquiátricos de Israel, os estudiosos descobriram índices altos de fumantes, $48,1 \%$ de técnicos e $76,0 \%$ de doentes ${ }^{(11)}$. De acordo com os achados, os autores concluíram que uma pessoa internada em um dos hospitais estudados é significativamente exposta a "atmosfera pró-fumo" e às conseqüências do fumo sobre sua saúde. Diante disto, fica um alerta para os integrantes das equipes de assistência em saúde mental quanto ao seu próprio hábito de fumar.

Os enfermeiros de saúde mental, trabalhando em hospitais psiquiátricos devem se empenhar em aumentar a qualidade de vida daqueles que se encontram sob seus cuidados ${ }^{(38)}$. Para tanto, devem lembrar, também, de prestar assistência aos aspectos físicos além dos psicológicos. Exemplo disto é mostrado por um grupo de pesquisadores $^{(39)}$ ao se referirem aos cuidados que a enfermagem deve ter com o problema de incontinência urinária dos usuários, geralmente os idosos, com exame físico para detectar possíveis causas passíveis de tratamento; assessoramento funcional, visando a participação da pessoa doente no cuidado da continência; avaliar a mobilidade da pessoa e suas habilidades relativas às atividades da vida diária; avaliação da estrutura física que cerca o usuário, como visibilidade, localização e estrutura do banheiro que pode servir tanto para melhorar como piorar o controle de urina.

\section{CONSIDERAÇÕES FINAIS}

Atualmente, no Brasil, temos recebido como científica, verdadeira e inquestionável a mensagem da desospitalização. Inclusive, a política oficial se encontra dominada e dissemina tais idéias pelos meios de comunicação. Como uma primeira crítica a este sistema, lembramos que os movimentos mundiais guardam em 
si próprios, diferenças e tendem a respeitar a realidade de cada localidade. A psiquiatria democrática italiana surgida com o movimento de desinstitucionalização tem sua aplicação e resultados já comprovados como positivos em seu berço, a Itália. 0 próprio movimento italiano não é sinônimo de desospitalização, mas de uma atitude que transcende a isto, que busca o direito de qualquer pessoa a viver em comunidade. Objetiva a cidadania para os doentes mentais, negligenciados, marginalizados, com sua conseqüente participação novamente junto aos demais integrantes da sociedade. Os autores e trabalhadores em saúde mental, atuantes na Itália, chamam a atenção para que não se copiem seus modelos de abordagem, mas que se criem modalidades de assistência próprias ao meio ao qual se destinem, e se continue aprendendo com o passar do tempo, nas vivências, como eles o fazem.

Em uma pesquisa de opinião ${ }^{(40)}$, a autora apresenta-se como professora de Serviço Social em Wisconsin e mãe de um filho esquizofrênico, defendendo a necessidade de asilo para alguns casos de grave comprometimento mental. Ela chama a atenção para o fato da nossa sociedade valorizar a autonomia, a independência e a liberdade e parecer não compreender pessoas que não conseguem tais ideais. No texto ela considera importantes e fundamentais os programas de reinserção da pessoa portadora de distúrbio mental na comunidade, mas ressalta que isto esconde uma não aceitação ou mesmo um preconceito contra os que não se adaptam a estes programas (que provavelmente estão com melhor estrutura que os nossos, por se tratar de primeiro mundo). Muitos doentes só se sentem mais incapazes com o excesso de estímulos do ambiente familiar $e$

\section{REFERÊNCIAS BIBLIOGRÁFICAS}

1. Thompson KS. Re-inventing progressive community psychiatry: the use of history. Commun Ment Health J 1993; 29(6): 495-508.

2. Bastos 0 . Aspectos éticos da terapêutica psiquiátrica. J Bras Psiquiatria 1981; 30(1):93-100.

3. Assis MMA. Reflexões sobre as contradições dos modelos assistenciais psiquiátricos. Rev Baiana Enf 1993 abril; 6(1):72-82.

4. Jackson G, Gater R, Goldberg D, Tantam D, Loftus L, Taylor H. A new community mental health team based in primary care. $\mathrm{Br} J$ Psychiatry 1993; 162:375-84.

5. Bock T. Long-term Mental Iness in Germany. Int J Social Psychiatry 1994; 40(4):276-82.

6. Feis CL, Mowbray CT, Chamberlain PJ. Serving the chronic mental ill in State and Community Hospitals. Commun Ment Health J 1990; 26(3):221-32.

7. Sgambati ERV. Reinternação e rejeição familiar: um estudo com pacientes psiquiátricos. [dissertação]. Ribeirão Preto (SP): Escola de Enfermagem de Ribeirão Preto/USP; 1983. extra-muros, necessitando de altas doses de medicamentos e atenção diária para se manterem de forma precária fora de hospitais. A simples internação acalma pelo afastamento das exigências sociais, diminuindo a necessidade de medicação. As estatísticas de resposta às abordagens comunitárias, segundo a autora, não levam em consideração as pessoas. $O$ que fazer com as que não respondem às abordagens comunitárias? A autora prossegue fazendo uma análise do problema da família que vem sendo responsabilizada pela doença de um de seus integrantes. Ela ressalta que a família, pelo contrário, paga um caro ônus com a doença, e que são comuns aparecerem quadros psicopatológicos por estresse, sendo a depressão o mais comum deles (cita trabalhos sobre este tópico). Ao comentar sobre asilos, a autora salienta que defende um local de abrigo, com cuidados médicos e sociais, alimentação e, sobretudo, amabilidade.

O artigo citado acima é instigante e mostra a opinião de uma profissional, publicada em um periódico respeitado nos Estados Unidos. Serve, no mínimo, como um alerta. Leva-nos a fazer uma reflexão sobre o que estamos tentando ou deixando de fazer com pessoas severamente comprometidas. Faltam-nos, ainda, condições de avaliarmos melhor quem seriam os mais severamente comprometidos, bem como estudos sérios sobre a nossa realidade, sobre a nossa clientela, sobre as nossas possibilidades de intervenção. E se começássemos perguntando aos usuários, seus familiares e à comunidade sobre o que entendem e o que julgam ser necessário ou possível fazer no campo da doença mental crônica? Não seria este um recomeço melhor ?

8. Valencia M, Rasein ML, Otero BR, Ryan P. Descripcion de la conducta de los pacientes psiquiatricos hospitalizados. Salud Ment 1987; 10(3):81-9.

9. Guerro D, Gomez JA, Teran A, Poveda R. Evolución psicosocial de una población de psicoticos cronicos internados en un hospital psiquiatrico. Rev Psiquiat Medicina Barcelona 1990; 17(2):63-6.

10. Stein WW. El habito de coleccionismo en un hospital mental peruano. Rev Neuropsiquiatr 1991; 54(1/2):40-6.

11. Mester R, Toren P, Ben-Moshe $Y$, Weizman A. Survey of smoking habits and attitudes of patients and staff in psychiatric hospitals. Psychopathology 1993; 26(2):69-75.

12. Colson DB, Allen JG, Coyne L, Deering D, Jehl N, Kearns W, Spohn H. Profiles of Difficult Psychiatric Hospital Patients. Hosp Commun Psychiatry 1986; 37(7):720-4.

13. Thompson JW, Belcher JR, DeForge BR, Myers CP, Rosenstein MJ. Changing characteristics of schizophrenic patients admitted to state hospitals. Hosp Commun Psychiatry 1993; 44(3):231-5.

14. Gregoire PA. The phenomenology of psychiatric hospitalization: the patient's experience and expectations. Acta Psychiatr Scand 1990; 39:210-2. 
15. Lee $\mathrm{H}$, Coenen $\mathrm{A}$, Heim $\mathrm{K}$. Island living: the experience of Ioneliness in a psychiatric hospital. Appl Nurs Res 1994; 7(1):7-13.

16. Myers DH, Leahy A, Shoeb H, Ryder J. The patients view of life in a Psychiatric Hospital. Br J Psychiatry 1990; 156:853-60.

17. Lovell K. User satisfaction with inpatient mental health services. J Psychiatr Ment Health Nurs 1995; 2(3):143-50.

18. Norris MK, Kennedy CW. The view from within: how patients perceive the seclusion process. J Psychosocial Nurs 1992; 30(3):713.

19. Greenberg L, Fine SB, Cohen C, Larson K, Michaelson-Baily AM, Rubinton P. An interdiscinplinary psychoeducation program for schizophrenic patients and their families in an Acute Care Setting. Hosp Commun Psychiatry 1988; 39(3):277-82.

20. Goldman CR, Quinn FL. Effects of a patient education program in the tratment of schizophrenia. Hosp Commun Psychiatry 1988; 39(3):282-7.

21. Cavalcanti MT. O tratamento do paciente esquizofrênico crônico: uma revisão. J Bras Psiquiatria 1991; 40(2):53-63.

22. Bellack AS, Mueser KT. Psychosocial treatment for Schizophrenia. Schiz Bull 1993; 19(2):317-36.

23. Bridges $K$, Beresford $F$. The systematic review in primary care of patients with chronic psychotic illness. J Ment Health 1994; 3(4):50712.

24. Booker J, Blymyer D. Solution-oriented brief residential treatment with "chronic mental patients". J Systemic Therapies 1994; 13(4):5369.

25. Correa JO, Ingala EM, Justo SN. Tareas de rehabilitación en un servicio psiquiátrico. Acta Psiquiátr Psicol Am Lat 1987; 33(2):131-4. 26. Lombertie ER. Soins et rehabilitation des patients atteints de schizophrenie d'evolution defavorable. Anales Medico Psychologiques 1993; 151(4):343-6.

27. Palmer-Erbs VK, Anthony WA. Incorporating Psychiatric rehabilitation principles into mental health nursing: an opportunity to develop a full partnership among nurses, consumers, and families. J Psychosocial Nurs 1995; 33(3):36-44.
28. Gottheil E, Winkelmayer R, Smoyer P, Exline R. Characteristics of patients who are resistant to desinstitucionalization. Hosp Commun Psychiatry 1991; 42(7):745-8.

29. Dias IP. Terapia ocupacional para pacientes psicóticos internados: estratégia em duas etapas: grupos I e II. J Bras Psiquiatria 1990; 39(1):17-22.

30. Almeida MIN. Uma experiência de Terapia Ocupacional com paciente psicótico. Bol Psiquiatria 1991; 24(1/2):9-13.

31. Goodwin ME. Art-therapy in an interdisciplinary program: for longterm institucionalized mental patients. Am J Art Therapy 1983; 22:8792.

32. Bueno SMV. Contribuição ao estudo da aplicação do lazer no ambiente hospitalar. [dissertação]. Ribeirão Preto (SP): Escola de Enfermagem de Ribeirão Preto/USP; 1981.

33. Biley F. Complementary therapy: using music in hospital settings. Nurs Stand 1992; 6(35):37-9.

34. Coudreaut-Quinn EA, Emmons MA, Mcmorrow MJ. Addherence and Accuracy: self-medication during inpatient psychiatric treatment. J Psychosocial Nurs 1992; 30(12):32-6.

35. Zaslove MO, Beal M, Mckinney RE. Changes in behaviors of inpatients after a ban on the sale of caffeinated drinks. Hosp Commun Psychiatry 1991; 42(1):84-5.

36. Torrey EF. Continuous treatment teams in the care of chronic mentally ill. Hosp Commun Psychiatry 1986; 37(12):1243-7.

37. Cosgray RE, Davidhizar RE, Grostefon JD, Powell M, Wringeer $\mathrm{PH}$. A day in the life of an inpatient: an experimental game to promote empathy for individuals in a psychiatric hospital. Arch Psychiatr Nurs 1990; 4(6):354-9.

38. Callaghan P, Adams R. The contribution of quality of life to developing therapeutic nursing in special hospitals. J Psychiatr Ment Health Nurs 1994; 1(2):109-14.

39. Jirovec NM, Brink CA, Wells TJ. Nursing assessments in the inpatient geriatric population. Nurs Clin North Am 1988; 23(1):21930.

40. Wasow M. The need for asylum revisited. Hosp Commun Psychiatry 1993; 44(3): 207-8,22. 